\title{
A new control system for high-precision In-Gas Laser Ionization and Spectroscopy experiments at KU Leuven
}

\author{
K. Dockx*, T.E. Cocolios, R. Ferrer, C. Granados ${ }^{1}$, S. Kraemer, Yu. Kudryavtsev, S. Sels ${ }^{1}$, \\ P. Van den Bergh, P. Van Duppen, M. Verlinde, E. Verstraelen, A. Zadvornaya ${ }^{2}$ \\ KU Leuven, Instituut voor Kern- en Stralingsfysica, B-3001 Leuven, Belgium
}

\section{A R T I C L E I N F O}

\section{Keywords:}

Laser spectroscopy

In-Gas-Jet Laser Ionization and Spectroscopy

Data acquisition

\begin{abstract}
A B S T R A C T
A new automated control system is developed for the In-Gas Laser Ionization and Spectroscopy (IGLIS) laboratory at KU Leuven. The IGLIS Control System is capable of stabilizing a narrowband single-mode tunable diode laser with a standard deviation of $1.14 \mathrm{MHz}$. Furthermore, the system controls and synchronizes all data acquisition for multiple techniques from resonant laser spectroscopy in gas cell or in gas jet to atomic PlanarLaser Induced Fluorescence (PLIF) spectroscopy of copper atoms seeded in a supersonic gas jet. The IGLIS Control System is validated by measuring the hyperfine splitting parameters of the ground state transition at $327 \mathrm{~nm}$ in ${ }^{63} \mathrm{Cu}$.
\end{abstract}

\section{Introduction}

Over the past decades, resonance ionization laser ion sources have become the workhorse for the selective and efficient production of radioactive ion beams using the Isotope Separation On-Line (ISOL) technique [1]. The isotope and, in some cases even, isomer selectivity is a direct consequence of the unique and well-defined atomic energy levels. The laser frequencies allow stepwise excitation and ionization of the isotopes. Furthermore, the resonant laser ionization techniques provide access to atomic observables, such as ionization potential, isotope shift and hyperfine structure parameters, that enable the extraction of nuclear properties, e.g. nuclear spin, changes in mean-square nuclear charge radii and nuclear moments [2]. However, present utilization of these techniques, e.g. in-source and in-gas laser spectroscopy, cannot provide a spectral resolution below a few $\mathrm{GHz}$, for (super) heavy nuclei due to high Doppler, temperature and/or pressure broadening of the resonances.

A new implementation of the In-Gas Laser Ionization and Spectroscopy (IGLIS) technique, referred to as in-gas-jet laser ionization and spectroscopy method, has been proposed [3]. This method has the capabilities to reach a higher resolution and is less dependent on the chemical properties of the element. In this technique, the produced isotopes are thermalized in a buffer gas cell, typically filled with a noble gas. The isotopes are transported by the gas to a de Laval nozzle at the gas cell exit that produces a supersonic jet, where stepwise laser ionization takes place. Due to the low pressure and low temperature in the gas jet, a high spectral resolution as low as $150 \mathrm{MHz}$ can be obtained, depending on the mass of the isotope of interest and the atomic excitation scheme used [4]. Therefore, the necessary data can be extracted to deduce atomic and nuclear properties of isotopes in the actinide, superheavy (transfermium) and the ${ }^{100} \mathrm{Sn}$ regions with sufficiently high precision to explore in detail their nuclear structure properties.

Performing high-resolution spectroscopy can only be achieved when experimental as well as external parameters are controlled or at least monitored. The laser frequency and laser power must be stabilized, while the timing for data acquisition of multiple systematic measurements is synchronized. Automated control systems can command these factors and contribute to an increased reliability and stability and general performance of the resonant laser ionization technique. A dedicated control system has been developed for the IGLIS laboratory at KU Leuven [5]. The IGLIS Control System is discussed in this article as a necessary component for present and future experiments to fully develop and optimize the in-gas-jet laser ionization and spectroscopy method, that will be implemented in the next generation radioactive ion beam facilities.

\footnotetext{
* Corresponding author.

E-mail address: dockx.kristof@kuleuven.be (K. Dockx).

${ }^{1}$ Current address: Engineering Department, CERN, CH-1211 Geneva 23, Switzerland.

${ }^{2}$ Current address: University of Jyväskylä, Department of Physics, P.O. Box 35, FI-40014 University of Jyväskylä, Finland.
} 


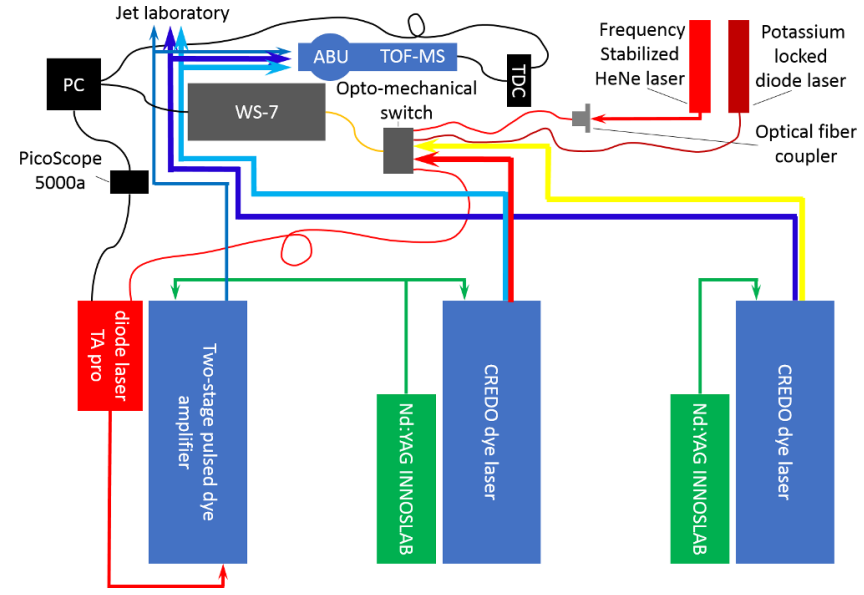

Fig. 1. Schematic layout of the laser system in the IGLIS laser laboratory. Image not to scale.

\section{The IGLIS laboratory}

The IGLIS laboratory in KU Leuven consists of a temperature-controlled $\left( \pm 0.5^{\circ} \mathrm{C}\right)$ ISO8 clean room that houses the laser system and a second room with a mass separator (IGLIS jet laboratory). The laser light is sent from the IGLIS laser laboratory to the IGLIS jet laboratory, where high-resolution in-gas-jet laser spectroscopy is performed.

\subsection{IGLIS laser laboratory}

The production of ion beams of a wide range of elements requires a versatile laser system. As every isotope has its element specific and unique resonant laser ionization scheme, the laser system must be capable of covering a large range of laser frequencies. A schematic layout of the complete IGLIS laser system is shown in Fig. 1. The laser system is equipped with two tunable dye lasers (CREDO, Sirah Lasertechnik $\mathrm{GmbH}$ ) and a pulsed dye amplifier (Sirah Lasertechnik $\mathrm{GmbH}$ ), all pumped by two Nd:YAG INNOSLAB lasers (Edgewave $\mathrm{GmbH}$ ). These two pump lasers can be operated at a maximum pulse repetition rate of $15 \mathrm{kHz}$ and generate frequency-doubled green light at $532 \mathrm{~nm}$ or frequency-tripled UV light at $355 \mathrm{~nm}$ with a maximum output power of $90 \mathrm{~W}$ or $36 \mathrm{~W}$, respectively.

The tunable dye lasers cover a fundamental wavelength range from 370 to $900 \mathrm{~nm}$. These lasers are equipped with two optical gratings in the laser resonator cavity with a groove density of 1800 lines $/ \mathrm{mm}$ and 3000 lines $/ \mathrm{mm}$. The optical gratings can be used in a laser wavelength range from 560 to $900 \mathrm{~nm}$ with a linewidth of $0.08 \mathrm{~cm}^{-1}$ at $570 \mathrm{~nm}$, equivalent to $2.4 \mathrm{GHz}$, while pumping with the $532 \mathrm{~nm}$ green laser light or a laser wavelength range from 370 to $560 \mathrm{~nm}$ with a linewidth of $0.05 \mathrm{~cm}^{-1}$ at $560 \mathrm{~nm}$, equivalent to $1.5 \mathrm{GHz}$, while pumping with the $355 \mathrm{~nm}$ blue laser light, respectively. In case one searches for unknown atomic transitions, the laser linewidth can be increased by a factor of four by using an intracavity prism telescope. Furthermore, second harmonic generation units are installed to cover a full wavelength range from 215 to $900 \mathrm{~nm}$.

For high-resolution spectroscopy purposes, narrowband laser radiation can be obtained by pulsed-dye amplification of a single mode continuous wave tunable laser covering a range from 649.9 to $657.1 \mathrm{~nm}$. The continuous wave laser light is produced by a TA pro diode laser (TOPTICA Photonics AG) with a specified linewidth below $1 \mathrm{MHz}$. This diode laser is controlled by Sys-DC-110 Diode Laser Driver Electronics (TOPTICA Photonics AG), equipped with a DCC-110 current control, a DTC-110 temperature control and a SC-110 scan control module. The laser light from the diode laser is sent to the pulsed dye amplifier (PDA). After single- or double-stage amplification in the PDA, a Fourier limited linewidth of $\sim 63 \mathrm{MHz}$ in the fundamental laser radiation at $654.8 \mathrm{~nm}$ is achieved as a result of the pump laser pulse with a time profile of $\sim 7 \mathrm{~ns}$. A frequency conversion unit with a BBO doubling crystal, specified for a wavelength range from 250 to $380 \mathrm{~nm}$, is installed in the PDA for second harmonic generation of the amplified single-mode and pulsed laser radiation.

Furthermore, two reference lasers are installed in the laboratory, a frequency-stabilized HeNe reference laser (Model 32734, Research Electro-Optics, Inc.) and a narrowband continuous wave diode laser system (DL pro 780, TOPTICA Photonics AG), which is frequency locked to a potassium cell (CoSy, TEM Messtechnik GmbH). The wavelength of all lasers is monitored with a wavelength meter (WS7-60, HighFinesse $\mathrm{GmbH}$ ) equipped with a four-channel opto-mechanical switch box (HighFinesse GmbH). The switch box makes it possible to monitor up to four different lasers simultaneously. A pulse generator (9530 Series Quantum Composers Inc.) triggers the two pump lasers and, therefore, controls the frequency and delay (250 ps timing and $<50 \mathrm{ps}$ jitter) of the different laser pulses for temporal and spatial overlap in the targeted ionization region.

Finally, the laboratory is equipped with an Atomic Beam Unit $(\mathrm{ABU})$. In the $\mathrm{ABU}$, one can perform high-resolution spectroscopy and time-of-flight mass spectrometry measurements. This device is used as a reference unit for resonant laser spectroscopy on stable isotopes. An atomic vapor is excited and, subsequently, ionized by resonant laser radiation from the PDA and the broadband tunable dye laser, respectively. The ionized isotopes are accelerated via two electrodes in the ABU towards a multichannel plate (MCP), where the isotopes are detected. The acquired signals from the MCP detector are digitized by a time-to-digital converter (TDC) with 512 channels and a time resolution of $4 \mathrm{~ns}$. The TDC is triggered by the laser pulse, therefore, one performs mass separation of the ionized isotopes based on their time-of-flight to the MCP detector [6].

\subsection{IGLIS jet laboratory}

For in-gas-jet laser resonant ionization and spectroscopy or atomic Planar-Laser Induced Fluorescence (PLIF) spectroscopy, the laser beams from the laser room are transported to the IGLIS jet laboratory [7]. This laboratory comprises an electrically grounded section and a high voltage section as shown in Fig. 2. In the grounded section, a vacuum chamber houses a gas cell equipped with a convergent-divergent (de Laval) nozzle for the production of a supersonic gas jet. A set of radiofrequency quadrupole (RFQ) ion guides transport the ionized isotopes up to the extraction electrode, where the isotopes are accelerated over a $40 \mathrm{kV}$ electric potential [8]. The formed ion beam is mass separated in a dipole magnet and observed in a detector station by either a multichannel plate or a Faraday cup (FC).

The laser radiation from the IGLIS laser laboratory can be directed in a longitudinal, transversal or combined orientation with respect to the supersonic gas jet or inside the gas cell. As such, one can perform ingas-cell or in-gas-jet resonant laser ionization and spectroscopy.

In addition, a laser beam can be shaped into a planar beam to overlap with the full gas jet in a perpendicular orientation. This configuration is required for atomic PLIF spectroscopy. The resonant laser

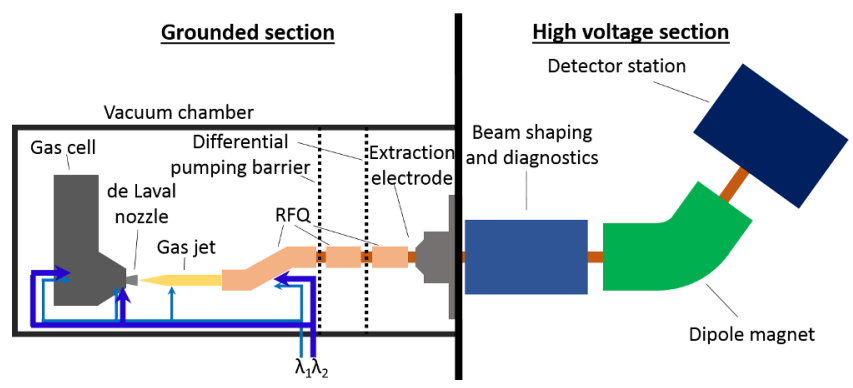

Fig. 2. Schematic layout of the IGLIS jet laboratory. Image not to scale. 
excited isotopes emit fluorescent photons by radiative de-excitation. These photons are detected by an iStar ICCD camera (ANDORDH334T). The accumulated images are stored and can be used for pixelby-pixel analysis of the gas jet structure, to extract possible density, temperature and velocity fluctuations over the full gas jet [7]. In its current configuration, the pixel size of $13 \mu \mathrm{m}^{2}$ corresponds to a spatial resolution of the gas jet of about $0.2 \mathrm{~mm}$. The photocathode in the camera can be externally triggered to maximize the signal-to-noise ratio, while multiple laser pulses can be recorded before extracting the pixel data. Optical filters in front of the lens attenuate scattered laser light and photons from direct de-excitation to the ground state to further increase the signal-to-noise ratio. For the same reason, residual background light is subtracted by image processing [7].

\section{IGLIS Control System}

\subsection{Requirements}

High-resolution resonant laser spectroscopy can only be performed when the laser light has a stable and tunable wavelength. Ideally, the spectral resolution is Doppler limited, i.e. $\sim 100 \mathrm{MHz}$ for in-gas-jet resonant laser ionization at high Mach numbers [3]. Therefore, the laser linewidth should be smaller than or similar to the Doppler broadening of the atoms in the jet to avoid a significant spectral broadening effect from the laser radiation, while keeping a maximum ionization efficiency. In addition, the timing between different laser pulses is crucial to resonantly ionize the atoms and has a distinct influence on the resonance linewidth as well [9]. One final requirement is the remote operation and control of the apparatus. While stabilizing and controlling the laser system, the control software should operate the different devices to ensure synchronized data taking. All of this will contribute to a higher resolution and an increased signal-to-noise ratio for the spectroscopic data, together with an increased reproducibility of the measurements.

The above requirements can be satisfied by controlling and/or reading a set of devices. The wavelength meter and the TA pro seeding laser allow the laser wavelength and its stability to be controlled. Control over the temporal and spatial overlap of the different laser pulses is possible via the pulse generators. Synchronization of the data taking can be performed by centralizing all acquisitions.

\subsection{Structure of the IGLIS Control System}

To meet all of the above requirements, a modular three-layered software system is developed, referred to as the IGLIS Control System. The full system has been implemented using the Xojo software framework (Xojo Inc.), because of its drag-and-drop user interface for simple graphical user interface development. Furthermore, the high level programming language simplifies and accelerates the full command and control implementation. A schematic overview of the program tree structure is shown in Fig. 3.

The software has a modular design, that allows a simple implementation of new devices and expansion of the control system for the acquisition of new data types. The bottom layer of the control system takes care of all direct device communication. Each device is controlled by a distinct module of the control system. Therefore, data communication with one device does not hamper other devices. When data is acquired, the information is collected by the device control module. Afterwards, the data streams upwards to the dataserver, the next layer in the IGLIS Control System. This layer is the core of the full control system and is responsible for collecting, sorting and saving all data from the device control modules. Moreover, part of this data flows further upstream to the top layer (IGLIS Mother Control). This part of the control system is the graphical user interface where the status of each device is shown, as well as the most important data. Furthermore, all settings for each of the devices are controlled through this IGLIS

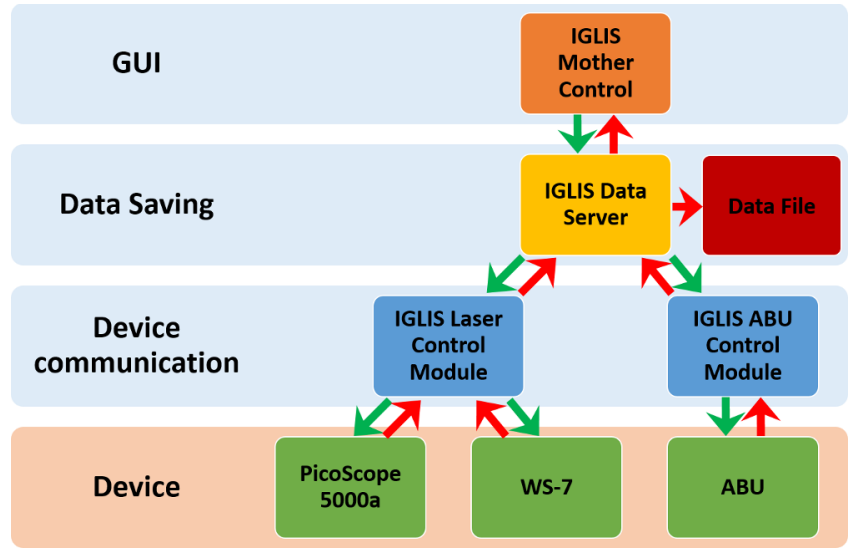

Fig. 3. Schematic overview of the tree structure in the IGLIS Control System. Acquired data flows upwards from the devices to the graphical user interface and is saved (red arrows). Setting information flows downwards from the graphical user interface to the appropriate devices (green arrows). (For interpretation of the references to colour in this figure legend, the reader is referred to the web version of this article.)

Mother Control. In preparation for a new measurement, settings for each of the desired acquisition devices are sent downstream to the dataserver and subsequently to the correct device.

Laser frequency selection and stabilization is performed by a proportional error correcting feedback loop with an open option to include an integral and derivative factor for a full PID correction loop. At a rate of up to $10 \mathrm{~Hz}$, limited by the exposure time for the wavelength meter measurements, the tunable diode laser wavelength is compared to a reference laser via the readout of their wavelengths in the wavelength meter. This reference laser can be the frequency-stabilized HeNe laser or the potassium-locked diode laser. In every iteration, the reference laser frequency readout from the wavelength meter is compared to its documented frequency. The obtained deviation from the documented frequency shows the wavelength meter drift. Similarly, the diode laser frequency is corrected to its setpoint taking into account the drift of the wavelength meter obtained from the reference laser readout.

As only one reference point is known from the HeNe or potassiumlocked diode laser, only zeroth order corrections for the wavelength meter drift can be applied, i.e. a wavelength independent offset; higher order drifts, that are wavelength dependent, cannot be corrected for. Present data show that this zeroth order correction limits the error at the stabilized wavelength to $1 \mathrm{MHz}$ for at least the first hour after calibration of the WS-7 wavelength meter [6]. Therefore, the zeroth order correction of the diode laser is performed by applying a DC voltage to the DC-110 diode laser driver via a pulse generator (PicoScope 5000a Pico Technology). This voltage is amplified in the DC-110 diode laser driver and applied to a piezo actuator in the diode laser for accurate control of the laser wavelength. When the diode laser is stabilized at the required wavelength, the desired data acquisition starts automatically via a pulse from the control software. When the preset amount of data is measured, communication between the appropriate device control module and the dataserver will tune the diode laser to the subsequent wavelength. Once the laser has stabilized at the new frequency, data acquisition will start again. This process continues until the full wavelength range is scanned and all data has been acquired. This typical diode laser scanning procedure, as used during spectroscopic measurements, is shown in Fig. 4.

\section{Applications and results}

The implementation of the IGLIS Control System has been extensively tested and used in three different spectroscopic techniques. The first method is resonant laser ionization in the ABU. Furthermore, 


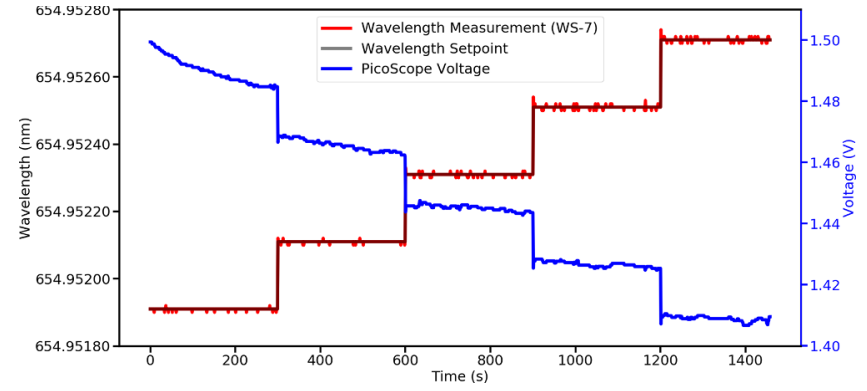

Fig. 4. Typical scanning procedure as performed during spectroscopic measurements. The diode laser wavelength is stabilized at preset values. Once data acquisition at one wavelength is finished, the wavelength will automatically be stabilized to the next wavelength setpoint. The red and black line (left scale) show the diode laser setpoint and measured wavelengths, respectively, as a function of time during a typical laser scan. The blue line (right scale) represents the correction voltage applied by the PicoScope to the diode laser driver to stabilize the diode laser wavelength at its setpoint value. (For interpretation of the references to colour in this figure legend, the reader is referred to the web version of this article.)

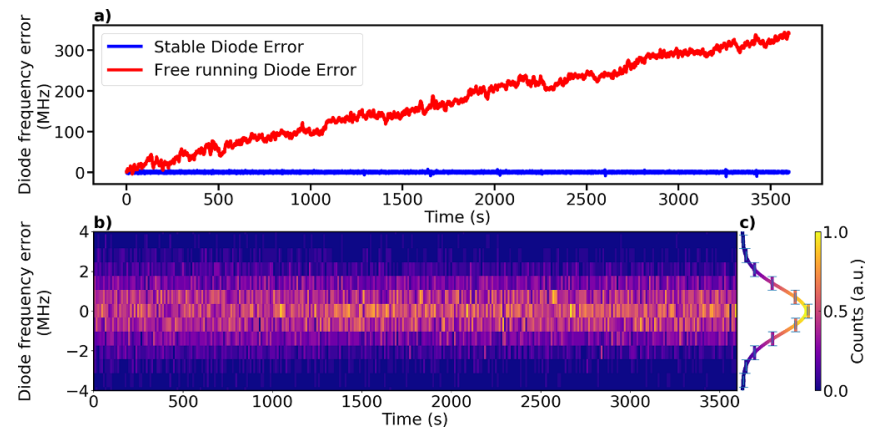

Fig. 5. Stability of the narrowband tunable diode laser over time. (a) The red line indicates the error between the setpoint diode frequency and the free running diode frequency over time. The blue line shows the error of the IGLIS Control System stabilized diode frequency including a zeroth order drift correction using a HeNe reference laser over time. (b) Density plot of the IGLIS Control System stabilized diode error as a function of time. (c) Normal distribution obtained after integration of the stabilized diode error over time. A standard deviation of $\sigma=1.14 \mathrm{MHz}$ is observed. (For interpretation of the references to colour in this figure legend, the reader is referred to the web version of this article.)

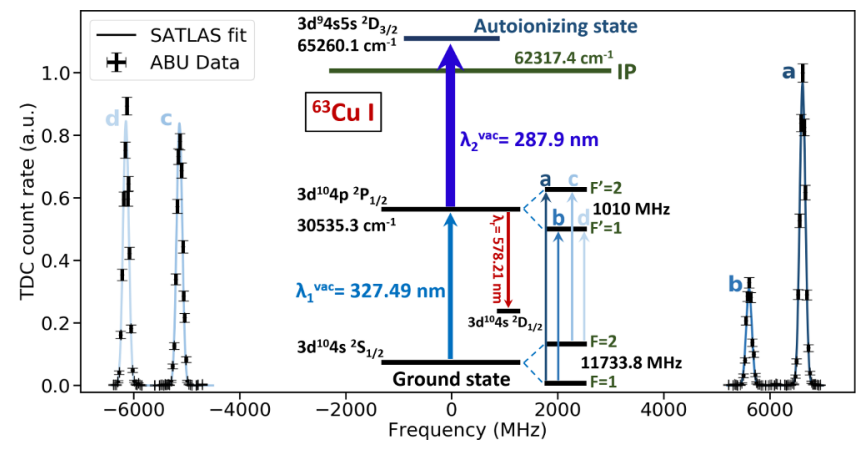

Fig. 6. Hyperfine structure of the $4 s^{2} S_{1 / 2}$ ground state to the $4 p{ }^{2} \mathrm{P}_{1 / 2}$ excited state in ${ }^{63} \mathrm{Cu}$ as measured in the atomic beam unit, relative to the center of mass of the full spectrum. Fitting with the SATLAS analysis package [11] gives a FWHM of $120 \mathrm{MHz}$ (blue lines). The inset shows the hyperfine splitting of both states of ${ }^{63} \mathrm{Cu}$ and the laser ionization scheme used. (For interpretation of the references to colour in this figure legend, the reader is referred to the web version of this article.)
Table 1

Hyperfine splitting $A$-parameters for the $4 s{ }^{2} \mathrm{~S}_{1 / 2}$ ground state and $4 \mathrm{p}{ }^{2} \mathrm{P}_{1 / 2}$ excited state in ${ }^{63} \mathrm{Cu}$ as extracted from high-resolution resonant laser spectroscopy in the $\mathrm{ABU}$ and in the gas jet.

\begin{tabular}{lll}
\hline & $\begin{array}{l}A_{63 \mathrm{Cu} 4 s}{ }^{2 \mathrm{~S}_{1 / 2}} \\
(\mathrm{MHz})\end{array}$ & $\begin{array}{l}A_{63} \mathrm{Cu} 4 \mathrm{p}^{2 \mathrm{P}_{1 / 2}} \\
(\mathrm{MHz})\end{array}$ \\
\hline Literature & $5866.90871(2)[12]$ & $505.2(8)[13]$ \\
Experimental ABU & $5866.8(5)$ & $\begin{array}{l}505.8(5) \\
\text { Experimental In-gas-jet }\end{array}$ \\
\hline
\end{tabular}

in-gas laser spectroscopy is performed in a gas cell or in a gas jet. Finally, one can perform atomic PLIF spectroscopy in the gas jet.

In this section, the improvement in laser stability is quantified and the IGLIS Control System is validated by high-resolution resonant laser spectroscopy in the $\mathrm{ABU}$ and using the in-gas-jet spectroscopy technique. Furthermore, the control system has been used for atomic PLIF spectroscopy for extensive characterization of the gas jet [7].

All presented measurements are performed on stable ${ }^{63,65} \mathrm{Cu}$ atoms, vaporized via resistive heating of a crucible filled with $\mathrm{Cu}$ foils in the $\mathrm{ABU}$ or direct resistive heating of a $\mathrm{Cu}$ filament in the gas cell. The $4 \mathrm{~s}$ ${ }^{2} \mathrm{~S}_{1 / 2}$ ground state to $4 \mathrm{p}{ }^{2} \mathrm{P}_{1 / 2}$ excited state transition of stable ${ }^{63,65} \mathrm{Cu}$ is probed in all of the following measurements by scanning the narrowband diode laser around $654.98 \mathrm{~nm}$, which is frequency doubled by a BBO conversion crystal in the PDA to $327.49 \mathrm{~nm}$ (in vacuum). In the case of resonant ionization spectroscopy in the ABU or in the gas jet, the excited isotopes are ionized via an autoionizing state with $287.9 \mathrm{~nm}$ laser radiation. This is provided by one of the broadband tunable dye lasers after frequency doubling the fundamental laser radiation with a wavelength of $575.8 \mathrm{~nm}$. In the atomic PLIF spectroscopy in the gas jet for stable ${ }^{63,65} \mathrm{Cu}$, radiative de-excitation occurs from the $4 \mathrm{p}^{2} \mathrm{P}_{1 / 2}$ excited state to the $4 s^{2}{ }^{2} \mathrm{D}_{3 / 2}$ metastable state with a branching ratio of $1.4 \%$ [10]. The emitted fluorescent photon has a wavelength of $578.21 \mathrm{~nm}$.

\subsection{Laser stabilization}

One of the main objectives for the IGLIS Control System is an improved and controllable wavelength stability of the scanning diode laser. Once the laser has reached a thermal equilibrium, the free running diode laser frequency drifts about $300 \mathrm{MHz}$ per hour from its setpoint frequency, see Fig. 5a. In addition, random fluctuations of about $50 \mathrm{MHz}$ are observed for the free running diode laser frequency. These instabilities are unacceptable, when one targets a resolution of $150 \mathrm{MHz}$. The implementation of the IGLIS Control System with live corrections on the wavelength readout, corrects for long term drifts in the laser wavelength due to either drifts in the wavelength meter or drifts from the diode itself. Fig. 5a shows the diode frequency difference from its setpoint frequency as a function of time for the unstabilized free running diode laser (red line) and the IGLIS Control System stabilized diode laser (blue line) using the HeNe reference laser. The random fluctuations for the stabilized diode laser have reduced significantly. A detailed density plot of the diode frequency error with the laser setpoint frequency as a function of time for the stabilized diode laser is shown in Fig. 5b. Integration in time yields the datapoints shown in Fig. 5c. As expected, a normal distribution is obtained. The corresponding standard deviation is found to be $1.14 \mathrm{MHz}$. As such, the IGLIS Control System reduces the random fluctuations in the diode laser by a factor of 50 . It can thus be concluded that the response function of the IGLIS Control System succesfully stabilizes the laser frequency. One has to be aware that an additional error on the diode laser frequency is introduced by the intrinsic stability of the frequency stabilized HeNe laser, i.e. $1 \mathrm{MHz}$ over $1 \mathrm{~h}$. Therefore, additionally including the earlier mentioned error due to wavelength dependent drifts in the wavelength meter, the upper limit for the precision of the narrowband tunable diode laser frequency can be calculated to be $1.8 \mathrm{MHz}$ for a one hour 
measurement. For measurements that take more than one hour, an automated hourly recalibration of the wavelength meter will offer a solution to ensure a $1.8 \mathrm{MHz}$ precision during the complete measurement. This feature is currently being developed and will be implemented in the IGLIS Control System in the near future.

\section{2. high-resolution spectroscopy in the $A B U$}

high-resolution resonant laser spectroscopy of stable ${ }^{63,65} \mathrm{Cu}$ is performed in the ABU. The data analysis allows to filter out the ${ }^{63} \mathrm{Cu}$ event from those originating from ${ }^{65} \mathrm{Cu}$ by means of time-of-flight separation. After applying corrections, that are independent of the control software and inherent to the wavelength meter, the spectrum shown in Fig. 6 is obtained for ${ }^{63} \mathrm{Cu}[6]$. The observed resonances are fitted using the SATLAS analysis package [11] and result in a FWHM of $120 \mathrm{MHz}$. The hyperfine splitting of both the ground and excited states are extracted as seen in Table 1 . The fitted hyperfine splitting $A$-parameters if found to be 5866.8(5) $\mathrm{MHz}$ and 505.8(5) MHz for the ground state and excited state splitting, respectively. These values are in perfect agreement with literature values. Therefore, one can conclude that no significant additional systematic error on the laser wavelength is introduced by the IGLIS Control System.

\subsection{In-gas-jet resonant laser spectroscopy}

In-gas-jet resonant laser spectroscopy is performed on stable ${ }^{63,65} \mathrm{Cu}$. The dipole magnet with a resolving power of $\mathrm{m} / \Delta \mathrm{m} \sim 300$ provides the mass separation necessary to obtain a pure single mass spectrum. The full hyperfine spectrum, similar to the spectrum in the $\mathrm{ABU}$, has been measured. A FWHM of $400 \mathrm{MHz}$ is extracted after fitting of each resonance. Once again, the fitted hyperfine splitting $A$-parameters agree with literature. As laser induced power broadening of the transitions is reduced to a minimum for this measurement, the transition width gives information on the Doppler broadening and, therefore, the Mach number of the gas jet and its temperature. Furthermore, from the Doppler shift, the element velocity in the gas jet can be extracted, from which the gas temperature in the gas cell can be determined as explained in [7]. Finally, the IGLIS Control System was used to perform atomic PLIF spectroscopy measurements. Information on the full jet was extracted and has been reported in [7].

\section{Conclusion}

The IGLIS laboratory at KU Leuven aims at performing in-gas-jet laser ionization and spectroscopy on the heavy elements with a resolution down to $150 \mathrm{MHz}$. This sets strict requirements on laser stability and reliability and to measuring devices. These requirements have been fulfilled by an automated control system, the IGLIS Control System. It is a remote-controlled modular program, capable of stabilizing a narrowband tunable diode laser frequency and synchronized data acquistion from IGLIS or atomic PLIF spectroscopy. The IGLIS Control System response function acts fast enough to control the frequency of the narrowband tunable diode laser within a standard deviation of $1.14 \mathrm{MHz}$. The corresponding total precision on the diode laser frequency is determined to be smaller than $1.8 \mathrm{MHz}$. The control system has been validated by high-resolution resonant laser spectroscopy in the ABU and using the in-gas-jet spectroscopy method, showing that no systematic error is introduced by the IGLIS Control System. Furthermore, continuous evolution of the system is simplified by the modular design of the software and can be performed without interfering with existing data acquisition.

\section{Acknowledgements}

This work has been funded by FWO-Vlaanderen (Belgium), by GOA/ 2015/010 (BOF KU Leuven), by the Interuniversity Attraction Poles Programme initiated by the Belgian Science Policy Office (BriX network P7/12), by a grant from the European Research Council (ERC-2011AdG-291561-HELIOS), and a KU Leuven START grant.

\section{References}

[1] V.N. Fedosseev, Y. Kudryavtsev, V.I. Mishin, Resonance laser ionization of atoms for nuclear physics, Phys. Scr. 85 (5) (2012) 058104, https://doi.org/10.1088/00318949/85/05/058104.

[2] P. Campbell, I. Moore, M. Pearson, Laser spectroscopy for nuclear structure physics, Prog. Part. Nucl. Phys. 86 (2016) 127-180, https://doi.org/10.1016/j.ppnp. 2015. 09.003.

[3] Y. Kudryavtsev, R. Ferrer, M. Huyse, P. Van den Bergh, P. Van, Duppen, The in-gasjet laser ion source: Resonance ionization spectroscopy of radioactive atoms in supersonic gas jets, Nucl. Instrum. Methods Phys. Res. Section B 297 (2013) 7-22, https://doi.org/10.1016/j.nimb.2012.12.008 arXiv:arXiv:1211.6649v1.

[4] R. Ferrer, A. Barzakh, B. Bastin, R. Beerwerth, M. Block, P. Creemers, H. Grawe, R. de Groote, P. Delahaye, X. Fléchard, S. Franchoo, S. Fritzsche, L.P. Gaffney, L. Ghys, W. Gins, C. Granados, R. Heinke, L. Hijazi, M. Huyse, T. Kron, Y. Kudryavtsev, M. Laatiaoui, N. Lecesne, M. Loiselet, F. Lutton, I.D. Moore, Y. Martínez, E. Mogilevskiy, P. Naubereit, J. Piot, S. Raeder, S. Rothe, H. Savajols, S. Sels, V. Sonnenschein, J.-C. Thomas, E. Traykov, C. Van Beveren, P. Van den Bergh, P. Van Duppen, K. Wendt, A. Zadvornaya, Towards high-resolution laser ionization spectroscopy of the heaviest elements in supersonic gas jet expansion, Nat. Commun. 8 (2017) 14520, https://doi.org/10.1038/ncomms14520.

[5] Y. Kudryavtsev, P. Creemers, R. Ferrer, C. Granados, L. Gaffney, M. Huyse, E. Mogilevskiy, S. Raeder, S. Sels, P. Van den Bergh, P. Van Duppen, A. Zadvornaya, A new in-gas-laser ionization and spectroscopy laboratory for off-line studies at KU Leuven, Nucl. Instrum. Methods Phys. Res., Sect. B 376 (2016) 345-352, https:// doi.org/10.1016/j.nimb.2016.02.040.

[6] M. Verlinde, K. Dockx, D. Studer, On the reliability of wavelength meters for medium- to high-resolution laser spectroscopy (2019).

[7] A. Zadvornaya, P. Creemers, K. Dockx, R. Ferrer, L.P. Gaffney, W. Gins, C. Granados, M. Huyse, Y. Kudryavtsev, M. Laatiaoui, E. Mogilevskiy, S. Raeder, S. Sels, P. Van den Bergh, P. Van Duppen, M. Verlinde, E. Verstraelen, M. Nabuurs, D. Reynaerts, P. Papadakis, Characterization of supersonic gas jets for high-resolution laser ionization spectroscopy of heavy elements, Phys. Rev. X 8 (4) (2018) 041008, https://doi.org/10.1103/PhysRevX.8.041008.

[8] S. Sels, Design and commissioning of an ion guide system for In-Gas Laser Ionization and Spectroscopy experiments, Nucl. Instrum. Methods Phys. Res., Sect. B (2019), https://doi.org/10.1016/j.nimb.2019.06.005.

[9] R.P. de Groote, M. Verlinde, V. Sonnenschein, K.T. Flanagan, I. Moore, G. Neyens, Efficient, high-resolution resonance laser ionization spectroscopy using weak transitions to long-lived excited states, Phys. Rev. A 95 (3) (2017) 032502, https:// doi.org/10.1103/PhysRevA.95.032502.

[10] R. Kurucz, B. Bell, Atomic Line Data Kurucz CD-ROM No.23, Cambridge Mass.: Smithsonian Astrophysical Observatory (1995). https://www.cfa.harvard.edu/ amp/ampdata/kurucz23/sekur.html.

[11] W. Gins, R. de Groote, M. Bissell, C. Granados Buitrago, R. Ferrer, K. Lynch, G. Neyens, S. Sels, Analysis of counting data: development of the SATLAS Python package, Comput. Phys. Commun. 222 (2018) 286-294, https://doi.org/10.1016/j cpc.2017.09.012.

[12] H. Figger, D. Schmidt, S. Penselin, La Structure Hyperfine Magnetique des Atomes et des Molecules, Colloques Internationaux du CNRS 164 (1967) 355.

[13] P. Vingerhoets, Nuclear structure of $\mathrm{Cu}$ isotopes studied with collinear laser spectroscopy, KU Leuven, 2011 Ph.D. thesis. 